\title{
The STAR detector upgrades and physics in beam energy scan phase II
}

\author{
Chi Yang ${ }^{1, a}$ for the STAR Collaboration \\ ${ }^{1}$ Key Laboratory of Particle Physics and Particle Irradiation (MOE) \\ Shandong University, Shanda South Road 27\#, Jinan, Shandong Province, China 250100
}

\begin{abstract}
The Beam Energy Scan Phase II at RHIC, BES-II, is scheduled from year 2019 to 2020 and will explore the high baryon density region of the QCD phase diagram with high precision. The program will focus on the interesting energy region determined from the results of BES-I. Some of the key measurements anticipated are: the net-protons kurtosis that could search for the critical point, the directed flow that might prove a softening of the EOS, and the chiral symmetry restoration in the dilepton channel. The measurements will be possible with the order of magnitude better statistics provided by the electron cooling upgrade of RHIC and with the detector upgrades planned to extend STAR experimental reach. The upgrades are: the inner TPC sectors (iTPC), the Event Plane Detector (EPD), and the end-cap TOF (eTOF). We present the BES-II program details and the physics opportunities enabled by these upgrades.
\end{abstract}

\section{Introduction}

The STAR Collaboration proposes a second phase of the Beam Energy Scan program at RHIC (BESII) to explore the QCD phase structure [1] from year 2019 to 2020. The Beam Energy Scan Phase I program was proceeded from year 2010 to 2014. From $\sqrt{s_{N N}}=200$ to $7.7 \mathrm{GeV}$ which are corresponding to baryon chemical potential $\mu_{B}=20$ to $420 \mathrm{MeV}, 8$ collision energies were scanned and many interesting results were achieved. At the top RHIC energy $\sqrt{s_{N N}}=200 \mathrm{GeV}$, the discovery of the quark-gluon plasma (QGP) is further confirmed. The results from BES-I identify the interesting collision energy range below $\sqrt{s_{N N}}=20 \mathrm{GeV}$. These results contain the studies related to the following topics: signature of the QGP turn-off $[2,3]$, the critical point $[4,5]$, the first-order phase transition [6] and chiral phase transition [7,8].

The BES-II program is consist with low-energy running, accelerator upgrade and detector upgrades. The program is focusing on precision measurements in the interesting energy region identified based on various BES-I results. The Au+Au collision data from $\sqrt{s_{N N}}=19.6,14.5,11.5,9.1$ and $7.7 \mathrm{GeV}$ will be collected in year 2019 and 2020. The detail event statistics for various observables related to the topics mentioned above can be found in Table 1. Compared to BES-I, the statistics will be improved by more than a factor of ten. To achieve these statistics requirements and obtain conclusive messages on these topics, accelerator upgrade at RHIC and detector upgrades at STAR are needed. At the same time, the Fixed-Target program (FXT) which uses only one beam allows us to

a e-mail: chiyang@sdu.edu.cn 
Table 1. Proposed event statistics (in millions) in Beam Energy Scan Phase-II compared to those in BES-I. The relations between collision energy and baryon chemical potential both in collision mode and fixed target mode are also shown in the table.

\begin{tabular}{c|c|c|c|c|c}
\hline \hline Collision Energy $(\mathrm{GeV})$ & 7.7 & 9.1 & 11.5 & 14.5 & 19.6 \\
$\mu_{B}(\mathrm{MeV})$ in 0-5\% central collisions & 420 & 370 & 315 & 260 & 205 \\
\hline Fixed Target Energy $(\mathrm{GeV})$ & 3.0 & 3.2 & 3.5 & 3.9 & 4.5 \\
Fixed Target $\mu_{B}(\mathrm{MeV})$ & 721 & 699 & 666 & 633 & 589 \\
\hline Proposed Event Goals in BES-II & 100 & 160 & 230 & 300 & 400 \\
BES-I Events & 4 & N/A & 12 & 20 & 36 \\
\hline
\end{tabular}

reach higher $\mu_{B}$ with high luminosity. The detail related between collision energy and FXT energy can also be found in Table 1 .

\section{Upgrades}

To make high statistics measurements of the observables, the operation luminosities of RHIC need to be increased. The low energy electron cooling program is ongoing at RHIC. This electron cooling system can raise the luminosity by a factor of $3 \sim 10$ in the range from $\sqrt{s_{N N}}=5$ to $20 \mathrm{GeV}$ while the longer bunches will increase luminosity by factor of $2 \sim 5$.

At the same time, three detector upgrades are ongoing at STAR. They are the iTPC upgrade [9], the EPD upgrade and the eTOF upgrade [10]. The major goals of the iTPC upgrade are as follows: wider rapidity coverage, better momentum resolution, and better $\mathrm{dE} / \mathrm{dx}$ resolution. By increasing the number of inner sector readout pad rows from 13 to 40 and increasing the pad size, we double the readout channels will get continuous coverage on $\eta$. This will increase the percentage of sampled track path length from $20 \%$ to $95 \%$ and extend the $|\eta|$ coverage from 1 to 1.5 . The electronics will be upgraded accordingly. In addition, the multi-wire proportional chambers (MWPCs) in inner sectors will be renewed to reset the time for wire aging on anode wires due to the increasing integrated and instantaeous luminosity delivered by RHIC. In general, the goal for the projects is to replace all 24 existing inner sectors in the STAR TPC with new, fully instrumented, sectors. Now the first new inner sector has been installed on STAR detector and will be commissioned in the coming RHIC run. For this sector, the local test on the MWPC showed qualified energy resolution (20\% FWHM) in P10 gas which can be found in FIG. 1.

The EPD is a dedicated event-plane and centrality detector placed in the forward rapidity region $2<|\eta|<4$. With 16 radial segments and 24 azimuthal segments, the detector will provide precise measurements of both the collision centrality and the event plane. Additionally, the EPD will be a good trigger detector for collisions at lower beam energies with good time resolution $(\sim 1 \mathrm{~ns})$. For the eTOF upgrade, CBM and STAR join collaboration and will install $10 \%$ of the full CBM TOF system on STAR which is a part of the FAIR Phase 0 program. The eTOF will cover the rapidity range from -1.1 to -1.6 . Combining with the iTPC upgrade, the eTOF upgrade will provide particle identification capability in forward direction at STAR which is crucial to the Fixed Target program at BES-II. 


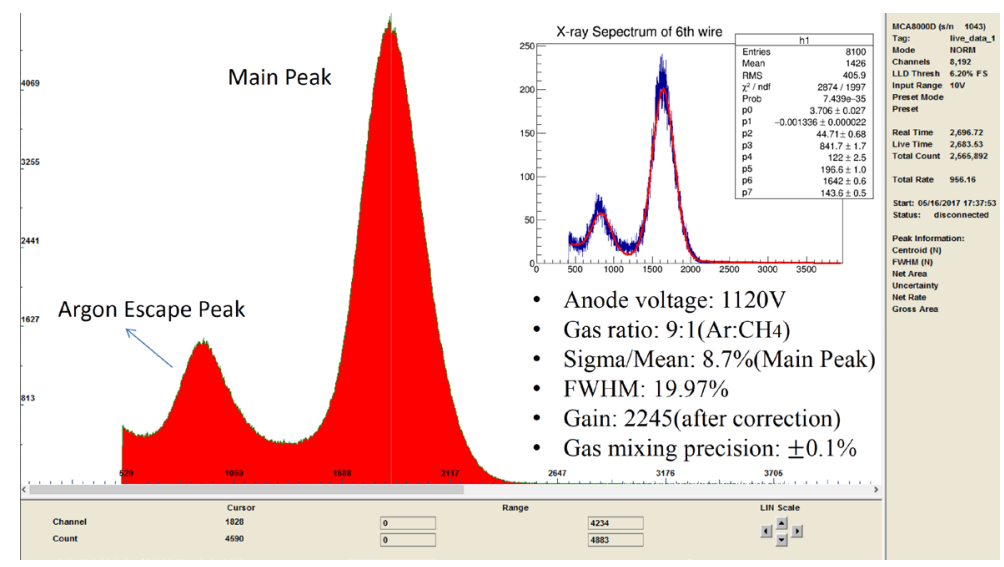

Figure 1. The spectrum of ${ }^{55} \mathrm{Fe}$ for the new inner TPC MWPC sector measured by MCA. The X axis represents the channel number, while $\mathrm{Y}$ axis represents the counts. A function which is consist with two gaussian functions and an exponential function is used to fit the data and get the energy resolution.

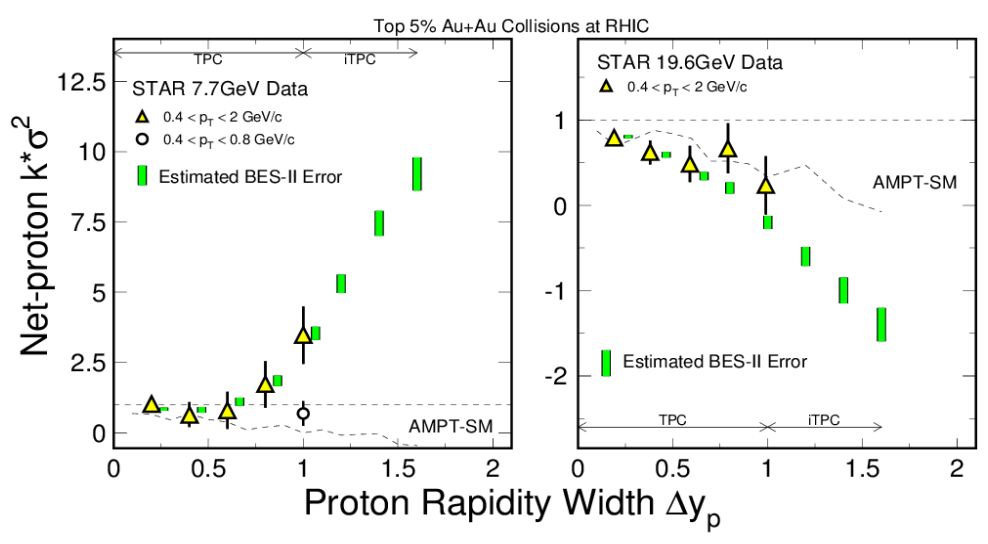

Figure 2. The net-proton kurtosis as a function of proton rapidity width. The yellow triangles represent the measurements in BES-I. The green boxes represent the uncertainty projection in BES-II. The projection mean values are based on data trend.

\section{Physics Opportunities}

With the performance improvements provided by collider and detector upgrades, many physics opportunities will be opened in BES-II. The significant improved statistics will benefit all statistics hungry analyses.

The iTPC upgrade will provide better rapidity and $p_{T}$ coverage. In BES-I, we observed sign change of $d v_{1} / d y$ for proton and net-proton at intermediate centralities below $20 \mathrm{GeV}$. The observed minimum for protons and net-protons resembles the predicted "softest point collapse" of flow and is a possible signature of a first-order phase transition between hadronic matter and a deconfined phase. In 
BES-II, the rapidity range of this direct flow measurement will be extended beyond the current midrapidity. This will expand our understanding of the role of baryon transport on the $v_{1}$ measurement. For net-proton cumulants measurement in BES-I, a non-trivial energy dependence has been revealed. The rapidity length of correlation in this measurement is important. As shown in FIG. 2, the green bands represents the uncertainty level achieved with the iTPC upgrade. The projected data points positions are based on the extended trend from the current measurement. This non-trivial energy dependence behavior can be studied and confirmed in a wider rapidity range with good statistics.

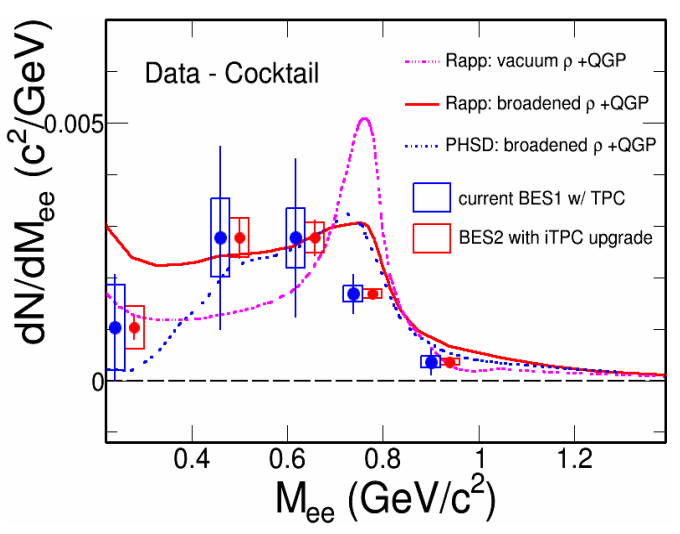

Figure 3. Dilepton uncertainty projection in the $\rho$ like region compared to the measurement in BES-I. The bars and boxes represent the statistical and systematic uncertainties, respectively. The projection mean values shown in red are set as same as current data.

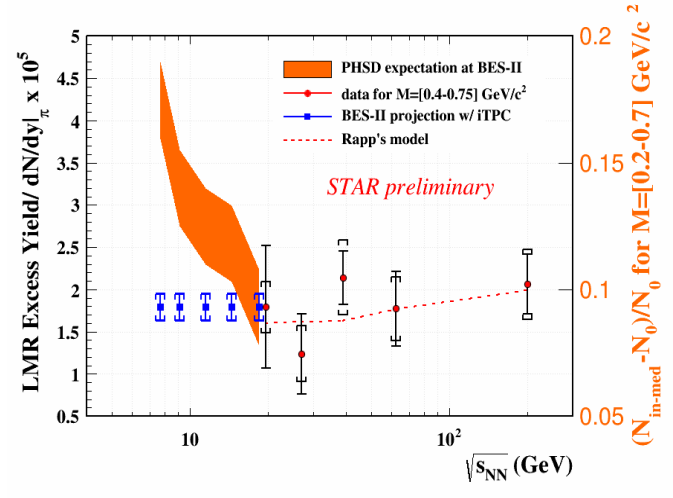

Figure 4. Normalized dilepton low mass excess as a function of collision energies. The blue markers represent the projection in BES-II with the iTPC upgrade. The projection mean values are set as same as current data at $19.6 \mathrm{GeV}$.

For dilepton measurements, we observed clear enhancement in the $\rho$-like region in BES-I. And these excesses are consistent with the $\rho$ broadening scenario within uncertainties which is based on partial chiral symmetry restoration in hot and dense medium [7, 8]. FIG. 3 shows the projected improvements from both statistical and systematic sides. As shown in FIG. 4, PHSD model predicts a "raising" trend of normalized low mass excess. In the figure, one can see that from 200 to $20 \mathrm{GeV}$ our measurements are consistent with the theoretical calculations and showed a ?flat? distribution within uncertainties. since in this range the total baryon density does not change much, it is important to extend this analysis to energy region below $20 \mathrm{GeV}$ where the total baryon density increases significantly as collision energy decreases. The blue markers give the projection precision below $20 \mathrm{GeV}$ in BES-II and the mean values of this projection are fixed to measured $19.6 \mathrm{GeV}$ mean value. Since iTPC combined with eTOF can provide forward EID capability, the dilepton analysis can be extended to forward rapidity in BES-II.

For the chiral magnetic effect (CME) measurement, the BES-I result shows a rapidly decreasing trend to 0 in the interval between 19.6 and $7.7 \mathrm{GeV}$ [2]. This may be caused by the probable domination of hadronic interactions over partonic ones. As one can see in FIG. 5, this dropping is with large uncertainty while the error projection in BES-II shown in pink box is much smaller.

The centrality definition and event plane resolution are important in BES-II. The EPD upgrade will benefit many analyses like net-proton higher moments, direct flow for net-protons, $v_{2}$ of identified particles and global $\Lambda$ polarization. In FIG. 6 , the red boxes represent the projected uncertainty of 
$d v_{1} / d y$ for net-proton in a narrow centrality gap 10-15\%. EPD will make this measurement possible while the current data give the uncertainty level like blue boxes in the figure.

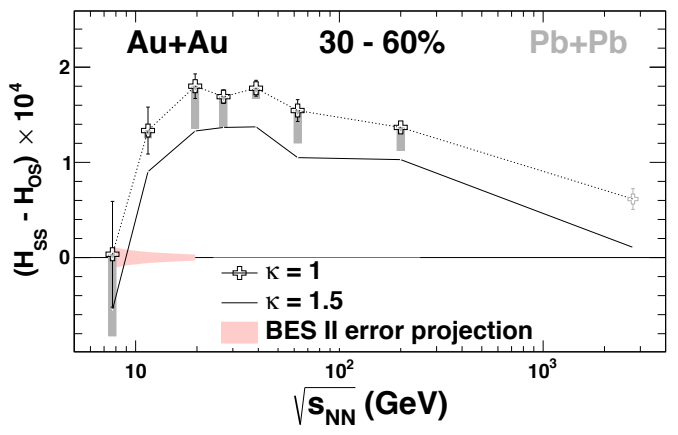

Figure 5. CME measured in BES-I compared to its BES-II error projection shown in pink box.

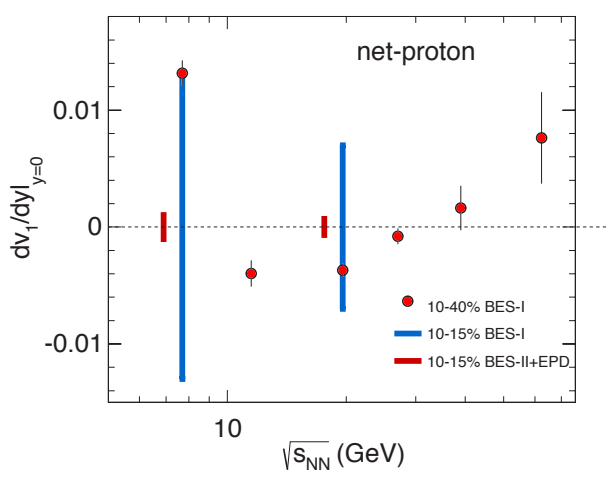

Figure 6. The error projection of $d v_{1} / d y$ measurement for net-proton in BES-II. The red boxes represent the BES-II uncertainty projection with the EPD upgrade while the blue boxes shows the current uncertainty in $10-15 \%$ centrality.

\section{Summary}

STAR proposes beam energy scan phase II in year 2019 and 2020 to explore the QCD phase diagram in high baryon density region. Many interesting topics will be further studied including the signature of the QGP turn-off, the critical point, the first-order phase transition and chiral phase transition. The upgrades from both RHIC (electron cooling system) and STAR (iTPC, EPD, eTOF) are ongoing and will be ready for BES-II data taken. With these upgrades, there will be significant improvements for the analyses at STAR: better statistics, lower systematic uncertainties, wider $p_{T}$ and rapidity coverage, and extended PID capability. The Fixed Target program enables high statistics studies below $7.7 \mathrm{GeV}$.

\section{Acknowledgement}

Supported by the National Natural Science Foundation of China (Grant Number: 11505181 and 11520101004).

\section{References}

[1] STAR Collaboration, STAR Note 598, https://drupal.star.bnl.gov/STAR/starnotes/public/sn0598

[2] L. Adamczyk et al. [STAR Collaboration], Phys. Rev. Lett. 113, 052302 (2014)

[3] L. Adamczyk et al. [STAR Collaboration], Phys. Rev. Lett. 110, 142301 (2013)

[4] L. Adamczyk et al. [STAR Collaboration], Phys. Rev. Lett. 112, 032302 (2014);

[5] L. Adamczyk et al. [STAR Collaboration], Phys. Rev. Lett. 113, 092301 (2014) 
[6] L. Adamczyk et al. [STAR Collaboration], Phys. Rev. Lett. 112, 162301 (2014)

[7] L. Adamczyk et al. [STAR Collaboration], Phys. Rev. Lett. 113, 022301 (2014)

[8] L. Adamczyk et al. [STAR Collaboration], Phys. Lett. B 750, 64 (2015)

[9] STAR Collaboration, STAR Note 619, https://drupal.star.bnl.gov/STAR/starnotes/public/sn0619

[10] STAR Collaboration and CBM eTOF Group, arXiv:1609.05102 